\title{
Mycobacteria as a cause of infective exacerbation in bronchiectasis
}

\author{
Christopher H.S. Chan, Andrew K.C. Ho ${ }^{1}$, Raphael C.Y. Chan ${ }^{1}$, H. Cheung ${ }^{2}$ \\ and Augustine F.B. Cheng ${ }^{1}$
}

Departments of Medicine, ${ }^{1}$ Microbiology and ${ }^{2}$ Diagnostic Radiology and Organ Imaging, Chinese University of Hong Kong, Prince of Wales Hospital, Shatin, NT, Hong Kong

\begin{abstract}
Summary: In 91 patients with bronchiectasis seen over 6 years, a positive mycobacterial culture was obtained in 12 cases (13\%). The organisms isolated were Mycobacterium tuberculosis in nine cases, Mycobacterium avium in two cases and Mycobacterium tuberculosis and chelonei were obtained on separate occasions in one case. Computed tomography and/or bronchography showed that the bronchiectatic changes commonly involved the lower lobes and to a lesser extent, the middle and lingula lobes. In none of these 12 cases was tuberculosis strongly suspected on clinical or radiological grounds.

We conclude that mycobacterial infections are common in patients with bronchiectasis and sputum should be cultured for mycobacteria periodically in these patients. In doubtful cases, bronchoscopy may be helpful to obtain a positive mycobacterial culture.
\end{abstract}

\section{Introduction}

The bacteriology causing colonization in patients with bronchiectasis is well documented. ${ }^{1}$ However, mycobacterial infections complicating bronchiectasis is seldom appreciated. In a survey of 223 patients with cystic fibrosis, ${ }^{2}$ Mycobacterium tuberculosis was identified in sputum of three and atypical mycobacteria of four cases. On the other hand, there are only occasional reports of $\boldsymbol{M}$. tuberculosis ${ }^{3}$ and atypical mycobacteria ${ }^{4-7}$ isolated in cases of bronchiectasis unrelated to cystic fibrosis. The true incidence of such infection is uncertain.

Because symptoms of chronic cough, sputum production, haemoptysis and systemic upset are common in patients with bronchiectasis, development of active mycobacterial infection in these patients is not usually suspected as infective exacerbation of bronchiectasis. ${ }^{8}$ Furthermore, the existing chest radiograph abnormalities of bronchiectasis may render it difficult to assess new shadowing related to mycobacterial infection. Hence, the diagnosis of mycobacterial infection in these patients presents a diagnostic challenge. We therefore undertook a survey of the prevalence of mycobacterial infections in patients with bronchiectasis to assess the magnitude of this problem.

Correspondence: Christopher H.S. Chan, M.B., B.S., M.R.C.P.

Accepted: 2 June 1992

\section{Patients and methods}

The records of all patients with the diagnosis of bronchiectasis made at the Prince of Wales Hos tal between January 1985 and December 1990 werece reviewed. Patients recruited into the study had? symptoms of chronic productive cough and/or recurrent haemoptysis together with radiological s evidence of bronchiectasis. Asymptomatic patientso were excluded. A plain chest radiograph with typical ring and/or 'tramline' shadows was con- $\triangle$ sidered adequate for diagnosis. In patients with $\overrightarrow{\hat{O}}$ equivocal or normal plain chest radiographs, com- $\exists$ puted tomography ${ }^{9}$ and/or bronchogram was required to confirm the diagnosis of bronchiectasis.

The following information was extracted fromo the case records: possible aetiology of bronchiec 3 . tasis (including history of pertussis, measles or $\frac{0}{3}$ pneumonia in childhood), history of tuberculosis, duration of symptoms of bronchiectasis, lobes involved (plain chest radiograph, computed tomo- $₹$ graphy or bronchogram), predominant organism 음 isolated in sputum, mycobacteria cultured from $>$ sputum or bronchoscopy specimens and timing of such positive growths. A positive culture of N atypical mycobacteria was considered significant ${ }^{\circ}$ only if it had been confirmed on at least three $\widetilde{N}$ occasions. Response to anti-tuberculosis treatment $\mathbb{N}^{N}$ was noted.

\section{Results}

We identified 91 symptomatic patients $\left(32\right.$ male) $\stackrel{\frac{0}{\circ}}{\frac{0}{\oplus}}$ with radiological evidence of bronchiectasis. The $\stackrel{\odot}{\Omega}$ 
mean age of the patients was 50.4 years (range 18-81). The presumed causes of bronchiectasis are listed in Table I. A history of tuberculosis preceding symptoms of bronchiectasis was obtained from 17 patients $(19 \%)$. In seven cases, the onset of symptoms of bronchiectasis coincided with the diagnosis of tuberculosis and the symptoms persisted despite completion of anti-tuberculosis treatment. In the other ten patients, tuberculosis preceded the onset of symptoms of bronchiectasis by 5-29 years. Cystic fibrosis has not been diagnosed in local Chinese and hypogammaglobulinaemia and Kartagener's syndrome were not encountered in our series. In many cases $(69 \%)$, the cause of bronchiectasis was unknown.

The predominant bacteria cultured from sputa are mostly Haemophilus influenzae, Pseudomonas aeruginosa and Staphylococcus aureus. A positive mycobacterial culture was obtained from 12 patients (13\%) during follow-up and their characteristics are shown in Table II. Nine patients had a positive culture of $M$. tuberculosis. Two patients had $M$. avium and one had both $M$. tuberculosis

Table I Presumed causes of bronchiectasis

\begin{tabular}{lcr}
\hline Cause & Number of patients & $(\%)$ \\
\hline Tuberculosis & 17 & $(19)$ \\
Pneumonia & 6 & $(7)$ \\
Measles & 5 & $(5)$ \\
Pertussis & 0 & $(0)$ \\
Unknown & 63 & $(69)$ \\
Total & 91 & $(100)$ \\
\hline
\end{tabular}

and $M$. chelonei cultured from sputum on separate occasions. Only one patient with $M$. avium infection was smear positive for alcohol-acid fast bacteria. The reasons for taking mycobacterial cultures in these 12 patients were: increase in cough and sputum, haemoptysis and dyspnoea. A clinical diagnosis of tuberculosis was not strongly suspected in any of these cases.

Radiological examination in these 12 cases showed that the lower lobes were bronchiectatic in seven cases, right middle or lingula lobe in four cases. One case showed radiological abnormality in the left upper lobe and one case had bronchiectasis involving all the lobes (Table II). None of them showed cavitation on chest radiograph and in none of them was tuberculosis suspected on radiological grounds alone.

\section{Response to anti-TB treatment}

All the patients with positive culture of $M$. tuberculosis were given anti-tuberculosis treatment except one patient (case 12 in Table II) who died of respiratory failure before the mycobacterial culture result was available. One patient died of respiratory failure despite treatment. Of the remaining eight patients, two had marked improvement, four had moderate improvement and two had persistence of symptoms after anti-tuberculosis treatment. In the patient with both $M$. tuberculosis and $M$. chelonei detected on sputum culture, $M$. tuberculosis cleared but $M$. chelonei persisted after completion of treatment.

In the two patients with positive cultures of $M$. avium, one had been given anti-tuberculosis drugs

Table II Characteristics of 12 cases of bronchiectasis with positive mycobacterial culture detected during follow-up

\begin{tabular}{|c|c|c|c|c|c|c|}
\hline Patient & Sex/age & $\begin{array}{l}\text { Duration of } \\
\text { bronchiectasis } \\
\text { (years) }\end{array}$ & $\begin{array}{l}\text { Predominant lobes } \\
\text { involved (method } \\
\text { of diagnosis) }\end{array}$ & $\begin{array}{l}\text { History of } \\
\text { tuberculosis } \\
\text { (years) }\end{array}$ & $\begin{array}{c}\text { Mycobacterial } \\
\text { culture }\end{array}$ & $\begin{array}{c}\text { Duration of } \\
\text { bronchiectasis preceding } \\
\text { positive mycobacterial } \\
\text { culture (years) }\end{array}$ \\
\hline 1 & $F / 60$ & 11 & RML, Ling (BG) & Nil & M. avium & 7 \\
\hline 2 & $F / 65$ & 1 & Ling (CT) & Nil & M. $T B$ & 1 \\
\hline \multirow[t]{2}{*}{3} & $F / 42$ & 17 & RLL, LLL (CT) & Nil & M. TB & 14 \\
\hline & & & & & M. chelonei & 14 \\
\hline 4 & $F / 28$ & 16 & RLL, LLL (CT) & Nil & M. $T B$ & 13 \\
\hline 5 & $\mathrm{~F} / 37$ & 9 & $\operatorname{RLL}(\mathrm{BG}, \mathrm{CT})$ & Nil & M. $T B$ & 5 \\
\hline 6 & $F / 44$ & 4 & Ling (BG) & 9 & M. $T B$ & 1 \\
\hline 7 & $\mathrm{~F} / 58$ & 10 & LUL (BG, CT) & Nil & M. $T B$ & 6 \\
\hline 8 & $\mathrm{M} / 49$ & 8 & RLL, LLL (CT) & 13 & M. $T B$ & 5 \\
\hline 9 & $\mathrm{M} / 62$ & 3 & RML, RLL (CT) & 8 & M. TB & 3 \\
\hline 10 & $\mathrm{M} / 70$ & 6 & RLL, LLL (CT) & Nil & M. TB & 5 \\
\hline 11 & $\mathrm{~F} / 58$ & 6 & LLL (BG, CT) & Nil & M. avium & 1 \\
\hline 12 & $\mathrm{~F} / 51$ & 10 & All lobes (CT) & 14 & M. $T B$ & 9 \\
\hline
\end{tabular}

RML = right middle lobe; $L U L=$ left upper lobe; $L L L=$ left lower lobe; $C T=$ computed tomography; $R L L=$ right lower lobe; Ling = lingula lobe; $\mathrm{BG}=$ bronchogram; $M . T B=$ Mycobacterium tuberculosis . 
but she emigrated to Holland shortly afterwards and her response to treatment was unknown. The other patient was not treated and yet the mycobacteria were cleared from the sputum spontaneously.

\section{Discussion}

Pulmonary tuberculosis is a well-recognized cause of bronchiectasis. In our patients, a history of tuberculosis was obtained in 17 cases $(19 \%)$. This is higher than the prevalence of $1-11 \%$ reported in other series. ${ }^{10-12}$ However, in our 12 patients with a positive mycobacterial culture on follow-up, a history of tuberculosis was only obtained in four patients. In these four cases, it is likely that tuberculosis was the underlying cause of the bronchiectasis and it was subsequently reactivated. It is possible that the patients without a previous history had subclinical mycobacterial infection which caused the bronchiectasis and the diagnosis of tuberculosis was only revealed subsequently. Alternatively, the bronchiectasis was due to some other cause and these patients developed infective exacerbations with mycobacteria. We have demonstrated an association between bronchiectasis and tuberculosis, although it is difficult to tell which came first.

Radiological examinations in the 12 patients with positive mycobacterial culture showed that the bronchiectatic changes were maximal over the lower lobes and to a lesser extent, the middle and lingula lobes. These are not typical sites of involvement in pulmonary tuberculosis. A previous study ${ }^{3}$ suggested that in some patients, damage to dependent bronchi by a primary tuberculosis infection at a young age was followed by spontaneous healing and later infection occurred because of their dependent location and poor drainage. Whatever the mechanism, the results of our study suggest that routine mycobacterial culture should be obtained from patients with bronchiectasis regardless of the lobes involved. A similar policy has been adopted in patients with cystic fibrosis. ${ }^{2}$

In most cases, the mycobacterium was cultured from sputum. However, one patient (Case 2 in Table II) presented with productive cough for 9 months. Chest radiograph showed persistent shadowing in the lingula lobe which remained static despite antibiotic treatment. Sputum for mycobacterial culture was negative on repeated occasions.

\section{References}

1. Pang, J.A., Cheng, A., Chan, H.S., Poon, D. \& French, G. The bacteriology of bronchiectasis in Hong Kong investigated by protected catheter brush and bronchoalveolar lavage. Am Rev Respir Dis 1989, 139: 14-17.
Computed tomography showed bronchiectatic change in the lingula lobe. Bronchoscopy was performed and $M$. tuberculosis was cultured from $\stackrel{\varrho}{C}$ the bronchoalveolar lavage fluid. A previous study has also indicated that in some patients with $\stackrel{5}{\circ}$ bronchiectasis, the tuberculous infection may only응 be revealed at operation. ${ }^{3}$ Therefore it seems that in $\underline{\bar{O}}$ some patients with bronchiectasis, the associated $\frac{\bar{\rho}}{\frac{\sigma}{\sigma}}$ tuberculous infection may run an indolent course. $\mathbb{\varnothing}$ Alternatively, in these patients, bronchiectasis is the result of subclinical tuberculous infection. The pathological changes in bronchiectasis consisting $\stackrel{\overrightarrow{ }}{\circ}$ of bronchial wall thickening, chronic inflammation $\overrightarrow{\vec{\omega}}$ and lymphoid aggregations $s^{13,14}$ may represent a ${ }_{\sigma}$ partially effective natural defence mechanism to $\frac{\overrightarrow{0}}{8}$ localize the tuberculous infection. This proposition 3 . is supported by the fact that none of the ten cases with tuberculosis associated with bronchiectasis $\infty$ was smear positive, suggesting that the mycobac- of terial load was small and investigations like bron- $\%$ choalveolar lavage ${ }^{15}$ may be required to recover the $\&$ organism from the bronchial tree. In addition, 음 none of the cases had cavitating disease and none was acutely ill to suggest fulminant mycobacterial $z$ infection.

There is no doubt that a positive culture of $M$. tuberculosis always needs treatment. On the other hand, the pathogenic role of atypical mycobacter in a patient with bronchiectasis is less clear cut. may just be a colonization of the abnormal bronct and treatment may not always be necessary. One patient had $M$. avium cultured from sputum on three separate occasions and yet the infection cleared spontaneously without treatment. Another patient had $M$. chelonei isolated from sputum on repeated occasions over 4 years. Because this organism was resistant to all first line drugs, second-line anti-tuberculosis drugs were not prescribed, but her chest radiograph showed very little change over the years. We would suggest that atypical mycobacteria cultured in a patient with bronchiectasis only warrant treatment if there is evidence of radiological progression or tissue invasion as demonstrated by bronchial biopsy.

We conclude that mycobacterial infection is a common complication in patients with bronchiec- 을 tasis especially in tuberculosis-endemic areas. The $D$ clinical and radiological features in these patients may be atypical and we recommend periodic $N$ routine mycobacterial culture, especially during episodes of infective exacerbations.

2. Smith, M.J., Efthimiou, J., Hodson, M.E. \& Batten, J.C. Mycobacterial isolations in young adults with cystic fibrosis. Thorax 1984, 39: 369-375. 
3. Rosenzweig, D.Y. \& Stead, W.W. The role of tuberculosis and other forms of bronchopulmonary necrosis in the pathogenesis of bronchiectasis. Am Rev Respir Dis 1966, 963, 769-785.

4. Bates, J.H. A study of pulmonary disease associated with mycobacteria other than Mycobacterium tuberculosis: clinical characteristics. Am Rev Respir Dis 1967, 96, 1151-1157.

5. Wolinsky, E. Nontuberculous mycobacteria and associated diseases. Am Rev Respir Dis 1979, 119: 107-159.

6. Albelda, S.M., Kern, J.A., Marinelli, D.L. \& Miller, W.T. Expanding spectrum of pulmonary disease caused by nontuberculous mycobacteria. Radiology 1985, 157: 289-296.

7. Yew, W.W., Kwan, S.Y.L., Wong, P.C. \& Lee, J. Ofloxacin and imipenem in the treatment of Mycobacterium fortuitum and Mycobacterium chelonae lung infections. Tubercle 1990, 71: $131-133$.

8. Knowles, M.R., Gilligen, P. \& Boucher, R.C. Cystic fibrosis. In: Mandell, G.L., Douglas, R.G. \& Bennett, J.E. (eds) Principles and Practice of Infectious Diseases, 3rd ed. Churchill Livingstone, New York, 1990, pp. 579-582.

9. Pang, J.A., Hamilton-Wood, C. \& Metreweli, C. The value of computed tomography in the diagnosis and management of bronchiectasis. Clin Radiology 1989, 40: 40-44.
10. Konietzko, N.F.J., Carton, R.W. \& Leroy, E.P. Causes of death in patients with bronchiectasis. Am Rev Respir Dis 1969, 100: 85-88.

11. Ellis, D.A., Thornley, P.E., Wightman, A.J., Walker, M., Chalmers, J. \& Crofton, J.W. Present outlook in bronchiectasis: clinical and social study and review of factors influencing prognosis. Thorax 1981, 36: 659-664.

12. Barker, A.F. \& Bardana, E.J. Jr. Bronchiectasis: update of an orphan disease. Am Rev Respir Dis 1988, 137: 969-978.

13. Whitwell, F. A study of the pathology and pathogenesis of bronchiectasis. Thorax 1952, 7: 213-219.

14. Lapa, J.R., Silva, E., Jones, J.A.H., Cole, P.J. \& Poulter, L.W. The immunological component of the cellular inflammatory infiltrate in bronchiectasis. Thorax 1989, 44: 668673.

15. Chan, H.S., Sun, A.J.M. \& Hoheisel, G.B. Bronchoscopic aspiration and bronchoalveolar lavage in the diagnosis of sputum smear-negative pulmonary tuberculosis. Lung 1990 168: $215-220$. 